\section{BMJ Open Respiratory Research}

\title{
Alcohol drinking and cigarette smoking in relation to risk of active tuberculosis: prospective cohort study
}

\author{
Avril Zixin Soh, ${ }^{1}$ Cynthia Bin Eng Chee, ${ }^{2}$ Yee-Tang Wang, ${ }^{2}$ Jian-Min Yuan, ${ }^{3,4}$ \\ Woon-Puay Koh ${ }^{1,5}$
}

To cite: Soh AZ, Chee CBE, Wang Y-T, et al. Alcohol drinking and cigarette smoking in relation to risk of active tuberculosis: prospective cohort study. BMJ Open Resp Res 2017;4:e000247. doi:10.1136/ bmjresp-2017-000247

- Additional material is published online only. To view please visit the journal online (http://dx.doi.org/10.1136/ bmiresp-2017-000247).

Received 21 September 2017 Revised 26 September 2017 Accepted 27 September 2017

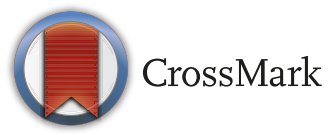

${ }^{1}$ Saw Swee Hock School of Public Health, National University of Singapore, Singapore, Singapore ${ }^{2}$ Singapore Tuberculosis Control Unit, Tan Tock Seng Hospital, Singapore, Singapore

${ }^{3}$ Division of Cancer Control and Population Sciences, University of Pittsburgh Cancer Institute, Pittsburgh, Pennsylvania, USA ${ }^{4}$ Department of Epidemiology, University of Pittsburgh Graduate School of Public Health, Pittsburgh, Pennsylvania, USA ${ }^{5}$ Office of Clinical Sciences, Duke-NUS Medical School, Singapore, Singapore

Correspondence to Dr Woon-Puay Koh, DukeNUS Medical School, Singapore, Singapore; woonpuay.koh@dukenus.edu.sg and Ms Avril Zixin Soh; avril.soh@u.nus.edu

\section{ABSTRACT}

Introduction Heavy alcohol consumption increases the risk of active tuberculosis (TB). However, the relation between lower levels of alcohol intake and TB risk remains unclear. We aimed to evaluate the association between alcohol intake and risk of active TB and assess whether the associations were modified by smoking status, which is another risk factor for active TB.

Methods The Singapore Chinese Health Study is a prospective cohort of 63257 adults aged 45-74 years recruited from 1993 to 1998. Information on alcohol intake and smoking history was collected at recruitment. Active TB cases were identified via linkage with National TB Notification Registry.

Results During a mean follow-up of 16.8 years, 1249 incident cases of active TB were identified. Among nonsmokers, compared with total abstinence, participants who had monthly to weekly intake of alcohol had reduced TB risk ( $\mathrm{HR} 0.70,95 \% \mathrm{Cl} 0.55$ to 0.89 ), but this reduction in risk with low-dose drinking was not observed among current smokers (HR 0.96, $95 \% \mathrm{Cl} 0.77$ to 1.18 ; $\mathrm{p}$ for interaction $=0.02$ ). Comparatively, drinking $2+$ drinks daily was associated with increased TB risk among current smokers (HR 1.51, 95\% $\mathrm{Cl} 1.11$ to 2.05). This increased risk was not observed among non-smokers (HR 0.93 $95 \% \mathrm{Cl} 0.49$ to 1.77 ) and the interaction between alcohol intake and smoking status was of borderline significance ( $p$ for interaction=0.08). In joint effect, compared with those who neither smoked nor drank, the risk of active TB increased from $1.82(95 \% \mathrm{Cl} 1.57$ to 2.10$)$ in current smokers who were non-drinkers to $3.16(95 \% \mathrm{Cl} 2.35$ to 4.24) in current smokers who also drank $2+$ drinks daily. Conclusion While low intake of alcohol may protect against active TB among non-smokers, drinking 2+ drinks daily could act synergistically with smoking to increase the risk of active TB in current smokers.

\section{INTRODUCTION}

Tuberculosis (TB) is caused by infection with Mycobacterium tuberculosis (Mtb) and is one of the leading causes of death worldwide from an infectious disease. ${ }^{1}$ It has been estimated that approximately 1.7 billion individuals in the world are latently infected with $\mathrm{Mtb},{ }^{2}$ but most infected individuals carry the Mtb bacillus in a clinically dormant state with no signs of active disease. ${ }^{3}$ Reactivation of latent infection occurs in approximately $10 \%$ of infected individuals, ${ }^{4}$ and the maintenance or reactivation of latent TB infection is dependent on the balance between host immune factors and bacillary growth. ${ }^{5}$ With the huge reservoir of latently infected individuals with risk of disease reactivation, identification of host factors that modulate the development of active TB is essential to support the development of effective TB control strategies in the population.

Heavy alcohol use or alcohol use disorders have been reported by two meta-analyses about a decade ago to be associated with approximately threefold increase in risk of developing active TB. ${ }^{6} 7$ Similarly, a recently published meta-analysis ${ }^{8}$ reported alcohol-related problems to be associated with about three times increase in risk of $\mathrm{TB}$, while alcohol use (vs no alcohol use) was also associated with increased TB risk, although with a smaller magnitude of effect (relative risk 1.35, $95 \%$ CI 1.09 to 1.68$).{ }^{8}$ While chronic alcohol exposure has been found to impair immune responses and the control of mycobacterial infection in many experimental and clinical studies, ${ }^{9-12}$ moderate intake of alcohol has also been reported to enhance immune responses in healthy adults ${ }^{13} 14$ and improve host control of mycobacterial infection in animal model. ${ }^{15}$ Hence, the relation between alcohol consumption and TB risk may be strongly dependent on the dose of exposure. Moderate consumption of alcohol has been extensively reported to protect against cardiovascular diseases, ${ }^{16}$ and dietary guidelines aimed to promote cardiovascular health have also included moderate alcohol intake as one of the components. ${ }^{1718}$ However, the effect of moderate alcohol intake on the risk of developing active TB, the leading cause of infectious disease mortality globally, has not been well examined. One of the meta-analyses ${ }^{6}$ had 
examined the relation between low alcohol exposure, defined as less than $40 \mathrm{~g}$ /day alcohol and risk of active TB and they did not find a significant association. However, only four studies on low alcohol exposure were included in their analysis and these studies were either conducted among patients who were cured of $\mathrm{TB}$ or retrospective case-control studies in which alcohol consumption could already be influenced by the development of TB disease. The more recently published meta-analysis ${ }^{8}$ also reported that a low alcohol intake of $\leq 24 \mathrm{~g} /$ day was not found to be associated with TB risk (RR 1.07, 95\% CI 0.93 to 1.22). The number of studies included in this analysis was also small (four studies), of which only two were prospective studies. Hence, with the small number of studies and wide range of alcohol intake levels encompassed within a single category for low alcohol exposure in these meta-analyses, ${ }^{6} 8$ the prospective association between lower intake levels of alcohol and the risk of active TB warrants further detailed investigations.

Furthermore, the examination of the alcohol-TB association in some of these studies ${ }^{1920}$ did not control for smoking status, which is a strong risk factor for active $\mathrm{TB} .{ }^{21}$ It is recognised that smoking and alcohol drinking are highly correlated lifestyle factors, independent of geography and race-ethnicity. ${ }^{22}{ }^{23}$ Thus, the relation between alcohol intake and TB risk could potentially be confounded by smoking status. In addition, both human and animal studies have shown that alcohol drinking and tobacco smoking could have potential synergistic effect due to the generation of common metabolites harmful to health. ${ }^{24}{ }^{25}$ Hence, the effect of alcohol on TB risk could be different between smokers and non-smokers.

Our main aim in this study was to investigate the association between alcohol intake and the risk of active TB in the Singapore Chinese Health Study (SCHS), a prospective population-based cohort of middle-aged to elderly adults in Singapore. Participants of this cohort went through periods when TB was highly prevalent in the country a few decades ago and those who had acquired latent TB infection in those early years would be at risk of disease reactivation at advanced age. ${ }^{26}$ To avoid confounding by smoking status, we studied the association separately among smokers and non-smokers in the stratified analysis. We also wanted to examine if the effect of alcohol could be different by smoking status in the stratified analysis to suggest that the alcohol-TB risk association may be modified by smoking.

\section{METHODS}

\section{Study population}

The SCHS is a population-based cohort of 63257 Chinese adults aged 45-74 years recruited between April 1993 and December $1998 .^{27}$ Participants were restricted to citizens or permanent residents of Singapore residing in government housing estates and they belonged to the two major dialect groups of Chinese in Singapore-the Hokkiens who originated from the southern part of Fujian Province and the Cantonese who came from the central region of Guangdong Province.

\section{Exposure assessment}

An in-person interview was conducted at the participant's home during recruitment and a structured questionnaire was used to collect information such as the participant's demographics, smoking history, height and weight and history of physician-diagnosed medical conditions such as diabetes and cancer. The body mass index (BMI) of the participant was calculated by dividing their weight in kilograms by height in metres squared. A 165-item semiquantitative food-frequency questionnaire specifically developed for this study population was used to assess the usual diet, including alcohol intake, of the participant over the past year.

We asked about the frequency and usual serving size for the intake of four different types of alcohol, that is, beer, wine, Chinese and Western hard liquor, in separate questions. For each type of alcohol, participants were asked to choose the response for their intake frequency from eight categories (never or hardly, once a month, 2-3 times a month, once a week, 2-3 times a week, 4-6 times a week, once a day and two or more times a day) and the usual serving size from four categories. The options for the serving size for beer included one small bottle $(375 \mathrm{~mL})$ or less, two small bottles or one large bottle $(750 \mathrm{~mL})$, two large bottles and three large bottles or more. For wine, the serving sizes were one glass $(118 \mathrm{~mL})$ or less, two, three and four glasses or more. For Chinese or Western hard liquor, the serving sizes were one shot $(30 \mathrm{~mL})$ or less, two, three and four shots or more. In our analysis, we defined one alcoholic drink as $375 \mathrm{~mL}$ of beer, $118 \mathrm{~mL}$ of wine and $30 \mathrm{~mL}$ of western or Chinese hard liquor. For each participant, the number of drinks consumed per day was computed from the product of the reported intake frequency and usual serving size. The smoking history of the participants was assessed by first asking whether they had ever smoked at least one cigarette a day for 1 year or longer. The response categories were 'No', 'Yes, but I quit smoking' and 'Yes, and I currently smoke' and these were used to classify the participants as never, former and current smokers, respectively. Former and current smokers also answered questions about their years of regular smoking $(9,10-19$, 20-29, 30-39 and 40+), number of cigarettes smoked per day (1-6, 7-12, 13-22, 23-32, 33-42 and 43+) and, for former smokers, years since smoking cessation $(<1,1-2$, $3-4,5-9,10-14,15-19$ and 20+).

\section{Case ascertainment and follow-up}

Cases of active TB among all participants of the cohort study were identified via linkage analysis of the cohort database with all records of the National TB Notification Registry $^{28}$ up to 31 December 2014. The notification of TB cases in the country is mandatory by law and all suspected and confirmed TB cases have to be notified to 
the Ministry of Health within 72 hours of starting TB treatment and/or laboratory-confirmed results. All patients with culture-positive TB in Singapore also are captured comprehensively in the National TB Notification Registry via electronic linkage with the two mycobacterial laboratories in Singapore. ${ }^{28}$ The vital status of the cohort participants was updated on an annual basis through record linkage analysis with all records of the Singapore Registry of Births and Deaths up to 31 December 2014. Cumulatively only 52 participants were known to be lost to the follow-up mainly due to migration out of Singapore.

\section{Statistical analysis}

Participants with a history of active TB before recruitment $(n=3012)$, as recorded in the National TB Notification Registry, were excluded from the analysis. Baseline characteristics of participants who developed TB and those who remained free of $\mathrm{TB}$ at the end of the study were compared using $\mathrm{X}^{2}$ test for categorical variables and Student's t-test for continuous variables. Personyears of follow-up for each participant were calculated from date of recruitment to date of diagnosis of active TB, death, lost-to-follow-up or 31 December 2014, whichever occurred earlier. We first examined alcohol intake and cigarette smoking in relation to TB risk in the whole study population before examining the alcohol-TB association with stratification by smoking status. Cox proportional hazards regression models were used to examine the relation between cigarette smoking and alcohol drinking and risk of developing active TB, with adjustments for factors that have been shown to affect TB risk in the literature or in our cohort. ${ }^{29-33}$ The categorisation of alcohol intake and cigarette smoking variables in the Cox models was based on cut-off values that were logical and convenient, that is, non-drinkers, monthly to weekly drinkers, daily drinkers of 1 drink/day and daily drinkers of 2+ drinks/day. Participants in the 'non-drinkers' category were those who reported intake of less than one drink per month. The proportional hazards assumption of the Cox models was assessed by including a time-dependent covariate for the variable in the model and there was no violation of the assumption (all $p$ Values $>0.44$ ). The strength of a given association was measured by the HR and its corresponding 95\% CIs. The models were adjusted for age at recruitment (years), year of baseline interview (1993-1995, 1996-1998), gender, dialect group (Hokkien, Cantonese), level of education (no formal education, primary school, secondary school or higher), BMI $\left(\mathrm{kg} / \mathrm{m}^{2}\right)$, total energy intake ( $\mathrm{kcal} /$ day), baseline history of diabetes (yes, no), tea intake (none, monthly, weekly, daily), energy-adjusted daily intake of protein, cholesterol, marine omega-3, omega- 6 fatty acids, vitamin $\mathrm{A}$ and vitamin $\mathrm{C}$ (quartiles), alcohol intake (none, monthly, weekly, daily one drink/day, daily 2+ drinks/ day) and smoking status (never, former, current). The highest level of education received by the study participant was our surrogate for socioeconomic status. We did not observe significant differences between men and women for the association between smoking status $(p$ for interaction $>0.11$ ) or alcohol intake categories ( $p$ for interaction $>0.16$ ) with TB risk. Furthermore, although most results were not statistically significant due to very small sample sizes for women who smoked or drank alcohol daily, the overall direction of the associations for drinking and smoking for women were similar to those for men (data not shown). Hence, the results for both genders were combined and presented with adjustment for gender in the statistical models.

To investigate the interaction between drinking and smoking in affecting active TB risk, we also examined the association between alcohol intake and TB risk with stratification by current smoking status (current smokers or non-smokers). Since former smokers were not found to have significant increased risk of active TB compared with never smokers, never and former smokers were combined into a single category of non-smokers in the stratified analyses. As the number of cigarettes smoked per day $(\leq 6,7-12,13-22,23-32,33-42,43+)$ and years of smoking ( $\leq 19$ years, 20-39 years, 40+years) among current smokers were found to be significantly associated with TB risk, these variables were also included in the same statistical model when we examined the alcohol-TB associations among current smokers. The heterogeneity of the associations by smoking status was tested by including an interaction term (product between alcohol intake categories and current smoking status) in the Cox model. Restricted cubic spline regression with four knots was used to test for non-linearity in the alcohol-TB associations by converting intake frequencies into average number of drinks/day as the exposure variable. The joint effect of alcohol intake and cigarette smoking was also examined by using participants who neither smoked nor drank as the reference group.

Analyses were conducted using SAS V.9.3 (SAS, Cary, North Carolina, USA) statistical software package except for the restricted cubic spline analysis, which was performed using Stata V.13 (Stata, College Station, Texas, USA). All the $p$ Values quoted were two-sided and $p<0.05$ was considered statistically significant.

\section{RESULTS}

During a mean follow-up of 16.8 (SD 5.2) years, 1249 incident cases of active TB were identified in our cohort. The incidence rates of $\mathrm{TB}$ within this cohort, adjusted to the age structure of the whole cohort, were 224 per 100000 person-years in men and 55 per 100000 personyears in women. The mean age at TB diagnosis was 68.7 (SD 9.1) years. Compared with participants who did not develop active TB at the end of the study, TB cases were older and more likely to be men. They were also more likely to have ever smoked and have a lower BMI and history of diabetes at recruitment (see online supplementary table 1). TB cases also reported higher daily energy intake, but lower daily energy-adjusted intake of 
protein, marine omega- 3 and omega- 6 fatty acids, vitamin $\mathrm{A}$ and vitamin $\mathrm{C}$ (see online supplementary table 1 ). The majority $(81.4 \%)$ of the cohort were non-drinkers of alcohol, defined as intake of less than one drink/month, while $7.2 \%, 8.0 \%$ and $3.4 \%$ of the cohort were monthly, weekly and daily drinkers, respectively. About $80.9 \%$ of the alcohol consumed in our cohort were from beer. Only $0.91 \%$ of our study participants reported alcohol intake of at least $40 \mathrm{~g} /$ day, which was the cut-off used to define heavy alcohol use in the meta-analysis. ${ }^{6}$ About $15.4 \%$ of non-drinkers in our cohort were current smokers while $53.1 \%$ of daily alcohol drinkers were current smokers. The percentage of daily alcohol drinkers among current smokers (9.5\%) was also higher than among non-smokers $(1.9 \%)$ (table 1). Overall, those who drank alcohol at least monthly were more likely to be men and have higher total energy intake (table 1). Daily alcohol drinkers had the lowest BMI and also the lowest energy-adjusted intakes of protein, marine omega-3, omega- 6 fatty acids, vitamin A and vitamin $\mathrm{C}$ compared with other categories of less frequent drinkers. The prevalence of diabetes was highest among non-drinkers (table 1).

Compared with never smokers, current smokers had higher risk of active TB (HR 2.07, 95\% CI 1.80 to 2.39) but there was no significant increase in TB risk among former smokers (HR 1.08, 95\% CI 0.89 to 1.30) (table 2). The risk of active TB among current smokers was also significantly higher when compared with former smokers (HR 1.90, 95\% CI 1.58 to 2.27). When we included both never and former smokers as a single category of non-smokers, current smokers had significantly increased risk of developing active TB (HR 2.02, 95\% CI 1.78 to 2.30 ). Among current smokers, the risk of active TB increased with increasing intensity and duration of cigarette smoking ( $p$ for trend $<0.001$ ) (table 2). Examination of TB risk among former smokers by the number of years they had quit smoking suggested that the risk of active TB decreased substantially within 2 years of smoking cessation (table 2).

When we examined the relation between alcohol intake and TB risk in the whole cohort, monthly to weekly drinkers had reduced risk (HR $0.83,95 \%$ CI 0.71 to 0.97 ), while daily drinkers with $2+$ drinks/day had higher risk of active TB (HR 1.45, 05\% CI 1.11 to 1.90) compared with non-drinkers (table 2). The association between alcohol intake and TB risk was also examined separately in non-smokers (combined never and former smokers together) and current smokers. Among non-smokers, risk of TB was significantly reduced for those who had monthly to weekly consumption of alcoholic drinks (HR $0.70,95 \%$ CI 0.55 to 0.89 ) compared with non-drinkers. However, such reduction in risk of TB with low level of alcohol consumption was not observed among current smokers (HR 0.96, 95\% CI 0.77 to 1.18; p for interaction $=0.02)$. In contrast, the consumption of 2 or more drinks daily was significantly associated with increased risk of active TB for current smokers (HR 1.51, 95\% CI 1.11 to 2.05 ) but not for non-smokers (HR 0.93 , 95\% CI
0.49 to 1.77 ) and the interaction between alcohol intake and smoking status was of borderline significance ( $\mathrm{p}$ for interaction $=0.08)($ table 3$)$.

We examined the joint effects of alcohol and cigarette use on risk of active TB. Compared with those who were neither current smokers nor drinkers of alcohol, the lowest risk was observed among non-smokers with monthly to weekly alcohol intake (HR $0.68,95 \%$ CI 0.54 to 0.86 ) (table 4). Conversely, the HRs (95\% CIs) of active TB increased to 1.82 (1.57 to 2.10) for current smokers who were non-drinkers and to 3.16 (2.35 to 4.24 ) for current smokers consuming 2 or more drinks/day (table 4).

We tested if the non-linearity in the association between average daily intake of alcoholic drinks and risk of active TB was significant by using the restricted cubic spline regression analysis with four knots. Among non-smokers, compared with non-drinkers, a J-shape relationship was observed; the risk of TB was significantly reduced between drinking at least once a month to less than daily, with the risk being lowest at intake about 1 drink/week (average of 0.13 drinks/day) and increasing toward null with higher intake (figure 1). The non-linearity in the association was statistically significant for non-smokers ( $p$ for non-linearity $=0.008$ ). In contrast, there was no evidence of a significant non-linear association between alcohol intake and TB risk for current smokers ( $p$ for non-linearity=0.52). Among current smokers, intake beyond approximately 2 drinks/day was associated with significantly increased risk of active $\mathrm{TB}$ compared with non-drinkers (figure 1).

Some subjects might have undiagnosed or unnoticed $\mathrm{TB}$ at the time of recruitment, which could affect the use of cigarettes and alcohol. To minimise the potential confounding effect of health and subclinical disease on the observed association, we repeated all analyses after excluding all deaths and TB cases that occurred within the first 2 years as well as the observed personyears of all participants during the first 2 years of follow-up. The results of these analyses on this reduced dataset were very similar to those based on the entire cohort. Compared with non-drinkers, HRs (95\% CIs) of developing active TB were 0.72 (0.56 to 0.93$)$ for those with monthly to weekly consumption of alcoholic beverage among non-smokers and 1.48 (1.06-2.06) for those consuming 2 or more drinks/day among current smokers.

Among 39528 participants contacted for a follow-up interview between 2006 and 2010, an average of 12.7 years after the baseline interview, $80.1 \%$ retained their drinking status as none, monthly, weekly or daily drinkers. Sensitivity analyses have been performed by examining the alcohol-TB associations only among participants who did not change their drinking status and we still observed similar trends in the results. Among non-smokers, the HR $(95 \% \mathrm{CI})$ for monthly to weekly drinkers was 0.47 (0.22 to 1.00) compared with non-drinkers and among smokers the HR $(95 \% \mathrm{CI})$ for those with $2+$ drinks/day was 1.46 ( 0.76 to 2.82$)$. 


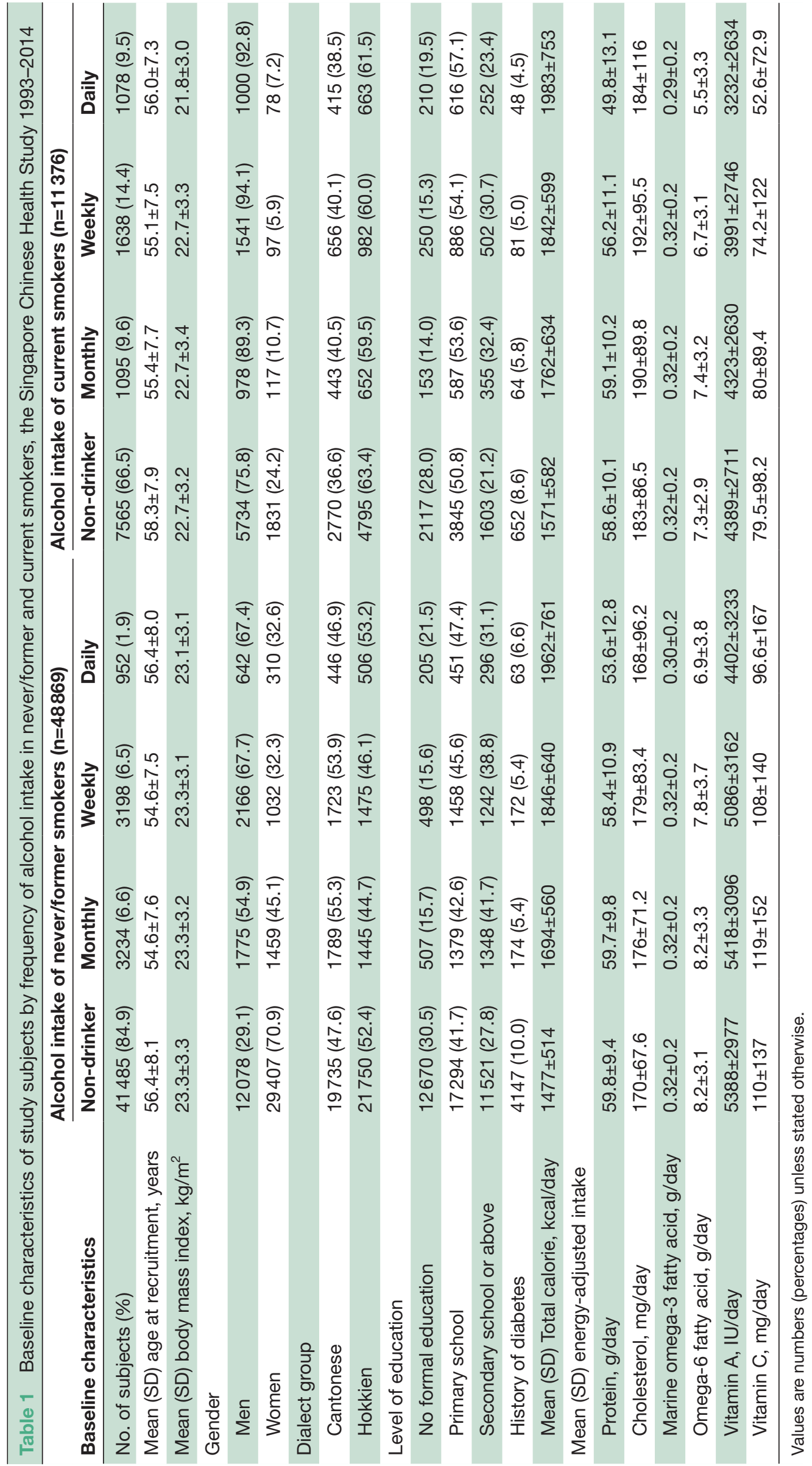


Table $2 \mathrm{HR}$ and $95 \% \mathrm{Cl}$ for alcohol intake and cigarette smoking at baseline in relation to risk of developing active tuberculosis, the Singapore Chinese Health Study 1993-2014

\begin{tabular}{|c|c|c|c|}
\hline & Cases & Person-years & HR $(95 \% \mathrm{Cl})^{*}$ \\
\hline \multicolumn{4}{|l|}{ Alcohol intake } \\
\hline Non-drinkers & 944 & 824265 & 1.00 \\
\hline Monthly to weekly & 208 & 156871 & 0.83 (0.71 to 0.97$)$ \\
\hline 1 drink/day & 30 & 13996 & 1.06 (0.73 to 1.53$)$ \\
\hline $2+$ drinks/day & 67 & 17558 & 1.45 (1.11 to 1.90$)$ \\
\hline \multicolumn{4}{|l|}{ Smoking status } \\
\hline Never smokers & 534 & 741538 & 1.00 \\
\hline Former smokers & 175 & 98290 & 1.08 (0.89 to 1.30$)$ \\
\hline Current smokers & 540 & 172861 & 2.07 (1.80 to 2.39$)$ \\
\hline \multicolumn{4}{|c|}{ Years of cigarette smoking among current smokers } \\
\hline Never smokers & 534 & 741538 & 1.00 \\
\hline$\leq 19$ years & 16 & 9951 & 1.76 (1.06 to 2.90$)$ \\
\hline 20-39years & 252 & 100335 & 2.02 (1.70 to 2.40$)$ \\
\hline $40+$ years & 272 & 62576 & 2.11 (1.77 to 2.51$)$ \\
\hline $\mathrm{p}$ Value for trend $\dagger$ & & & $<0.001$ \\
\hline \multicolumn{4}{|c|}{ Number of cigarettes/day among current smokers } \\
\hline Never smokers & 534 & 741538 & 1.00 \\
\hline$\leq 6$ & 55 & 27451 & 1.57 (1.18 to 2.09$)$ \\
\hline $7-12$ & 132 & 44931 & 1.97 (1.61 to 2.42 ) \\
\hline $13-22$ & 236 & 70387 & 2.19 (1.84 to 2.61$)$ \\
\hline 23-32 & 60 & 16107 & 2.22 (1.67 to 2.95$)$ \\
\hline $33-42$ & 38 & 9806 & 2.46 (1.75 to 3.48$)$ \\
\hline $43+$ & 19 & 4179 & 2.79 (1.74 to 4.48$)$ \\
\hline $\mathrm{p}$ Value for trend $\dagger$ & & & $<0.001$ \\
\hline \multicolumn{4}{|c|}{ Years of smoking cessation among former smokers } \\
\hline Never smokers & 534 & 741538 & 1.00 \\
\hline $10+$ years & 93 & 53851 & 1.04 (0.82 to 1.31$)$ \\
\hline 3-9years & 54 & 28002 & 1.17 (0.88 to 1.56$)$ \\
\hline$\leq 2$ years & 19 & 9140 & 1.24 (0.77 to 2.00 ) \\
\hline Current smokers & 540 & 172861 & 2.07 (1.80 to 2.38 ) \\
\hline $\mathrm{p}$ Value for trend $\dagger$ & & & $<0.001$ \\
\hline
\end{tabular}

*Adjusted for age at recruitment (years), year of baseline interview (1993-1995, 1996-1998), gender, dialect group (Hokkien, Cantonese), level of education (no formal education, primary school, secondary school or higher), body mass index $\left(\mathrm{kg} / \mathrm{m}^{2}\right)$, total energy intake ( $\left.\mathrm{kcal} / \mathrm{day}\right)$, baseline history of diabetes (yes, no), tea intake (none, monthly, weekly, daily), energy-adjusted daily intake of protein, cholesterol, marine omega-3, omega-6 fatty acids, vitamin A and vitamin C (quartiles), alcohol intake (none, monthly, weekly, daily 1 drink/day, daily $2+$ drinks/ day) and smoking status (never, former, current).

$\mathrm{tp}$ for linear trend tested by treating factor as continuous variable.

\section{DISCUSSION}

In our prospective cohort of middle-aged and elderly Chinese adults, low-dose intake of alcohol ranging from monthly to weekly was found to be associated with reduced risk of developing active TB compared with non-drinking, but this observation was only confined to non-smokers. Among current smokers, however, the consumption of 2 or more drinks daily significantly increased TB risk, but this increase in risk was not present in non-smokers. Examination of the joint effect of alcohol drinking and cigarette smoking showed that in comparison to individuals who were neither drinkers nor current smokers, daily intake of 2 or more drinks acted synergistically with smoking to increase the risk in current smokers, while non-smokers with less than 1 drink / day had the lowest risk of $\mathrm{TB}$.

The strengths of our study include the prospective study design which allowed us to establish the temporality of the associations, large sample size, long duration of follow-up and negligible loss to follow-up. The large 
Table $3 \mathrm{HR}$ and $95 \% \mathrm{Cl}$ for alcohol intake at baseline in relation to risk of developing active tuberculosis, stratified by smoking status, the Singapore Chinese Health Study 1993-2014

\begin{tabular}{|c|c|c|c|c|c|}
\hline \multirow[b]{2}{*}{ Alcohol intake } & \multicolumn{2}{|c|}{$\begin{array}{l}\text { Non-current smokers } \\
(\mathrm{n}=48869)\end{array}$} & \multicolumn{2}{|c|}{$\begin{array}{l}\text { Current smokers } \\
(n=11376)\end{array}$} & \multirow{2}{*}{$\begin{array}{l}p \text { Value for } \\
\text { interaction } \dagger\end{array}$} \\
\hline & Cases & HR $(95 \% \mathrm{Cl})^{*}$ & Cases & HR $(95 \% \mathrm{Cl})^{*}$ & \\
\hline Non-drinkers & 607 & 1.00 & 337 & 1.00 & \\
\hline Monthly to weekly & 84 & $0.70(0.55$ to 0.89$)$ & 124 & 0.96 (0.77 to 1.18$)$ & 0.02 \\
\hline 1 drink/day & 8 & $0.78(0.39$ to 1.58$)$ & 22 & $1.25(0.81$ to 1.93$)$ & 0.28 \\
\hline $2+$ drinks/day & 10 & $0.93(0.49$ to 1.77$)$ & 57 & $1.51(1.11$ to 2.05$)$ & 0.08 \\
\hline
\end{tabular}

*Adjusted for age at recruitment (years), year of baseline interview (1993-1995, 1996-1998), gender, dialect group (Hokkien, Cantonese), level of education (no formal education, primary school, secondary school or higher), body mass index ( $\left.\mathrm{kg} / \mathrm{m}^{2}\right)$, total energy intake $(\mathrm{kcal} / \mathrm{day})$, baseline history of diabetes (yes, no), tea intake (none, monthly, weekly, daily) and energy-adjusted daily intake of protein, cholesterol, marine omega-3, omega-6 fatty acids, vitamin A and vitamin C (quartiles), smoking status (never, former, current), cigarettes per day (non-current smokers, $\leq 6,7-12,13-22,23-32,33-42,43+$ ), years of smoking (non-current smokers, $\leq 19$ years, 20-39 years, 40+ years). $\mathrm{tp}$ for interaction was derived from the product term of smoking status and alcohol intake categories.

proportion of our study population with low to moderate levels of alcohol intake also enabled us to have a close examination of the relationship between lower levels of alcohol intake and TB risk. The ascertainment of active $\mathrm{TB}$ cases via linkage with the nationwide registry was virtually complete as the notification of TB cases is mandatory by law in Singapore. Even though Singapore currently has an intermediate TB incidence rate of about 40 per 100000 population, our study population consists of older residents who likely resided in the country when TB incidence was as high as 300 per 100000 population many decades ago. ${ }^{34}$ Hence, many of our participants could have acquired latent infection during those early years when TB was far more rampant ${ }^{26}$ and this makes our cohort suitable to study factors associated with reactivation of latent TB. We have previously reported how dietary factors such as lipids, vitamins and tea modulated the risk of active TB in this cohort. ${ }^{31-33}$ Hence, in this study that focused on the effect of alcohol consumption on active TB risk, we had included all these previously described factors as covariates in the model. Furthermore, since cigarette smoking is an established and strong risk factor of active $\mathrm{TB}$ and we had previously described the differential effects of vitamin $\mathrm{C}$ intake on the risk of active TB among smokers and non-smokers, ${ }^{32}$ we also examined for possible differential effects of alcohol consumption by smoking status in this study. We were also able to adjust for other factors which could be potential confounders in our analysis. ${ }^{29} 30$

Nevertheless, our study also has limitations which include assessment of the use of alcohol intake and smoking habits only at baseline. These factors could change over time. But as data were collected prospectively before the onset of disease, any subsequent changes would likely lead to non-differential misclassification and potential underestimation of the associations. As mentioned above, among 39528 participants contacted for a follow-up interview between 2006 and 2010 , an average of 12.7 years after the baseline interview, $80.1 \%$ retained their drinking status as none, monthly, weekly or daily drinkers. This advocates the stability of alcohol drinking behaviour in our study population. Further sensitivity analysis was performed by repeating the analysis among participants who did not change their drinking status at follow-up and the results for the alcohol-TB associations remained materially similar. We also did not know whether some of the participants in the 'non-drinkers' category could have been previous long-term alcohol consumers who had stopped drinking due to the development of health conditions. However, given the relatively low prevalence of drinking in this population, we do not expect this to be a major issue.

Table $4 \mathrm{HR}$ and $95 \% \mathrm{Cl}$ for joint effect of alcohol intake and cigarette smoking status in relation to risk of developing active tuberculosis, the Singapore Chinese Health Study 1993-2014

\begin{tabular}{|c|c|c|c|c|}
\hline \multirow[b]{2}{*}{ Alcohol intake } & \multicolumn{2}{|c|}{ Non-current smokers } & \multicolumn{2}{|c|}{ Current smokers } \\
\hline & Cases & HR $(95 \% \mathrm{Cl})^{*}$ & Cases & HR $(95 \% \mathrm{Cl})^{*}$ \\
\hline Non-drinkers & 607 & 1.00 & 337 & $1.82(1.57$ to 2.10$)$ \\
\hline Monthly to weekly & 84 & 0.68 (0.54 to 0.86$)$ & 124 & 1.81 (1.47 to 2.22$)$ \\
\hline 1 drink/day & 8 & 0.79 (0.39 to 1.59$)$ & 22 & $2.31(1.50$ to 3.56$)$ \\
\hline $2+$ drinks/day & 10 & $0.87(0.46$ to 1.64$)$ & 57 & $3.16(2.35$ to 4.24$)$ \\
\hline
\end{tabular}

*Adjusted for age at recruitment (years), year of baseline interview (1993 to 1995, 1996 to 1998), gender, dialect group (Hokkien, Cantonese), level of education (no formal education, primary school, secondary school or higher), body mass index $\left(\mathrm{kg} / \mathrm{m}^{2}\right)$, total energy intake $(\mathrm{kcal} / \mathrm{day})$, baseline history of diabetes (yes, no), tea intake (none, monthly, weekly, daily) and energy-adjusted daily intake of protein, cholesterol, marine omega-3, omega- 6 fatty acids, vitamin A and vitamin C (quartiles). 
Non-current smokers

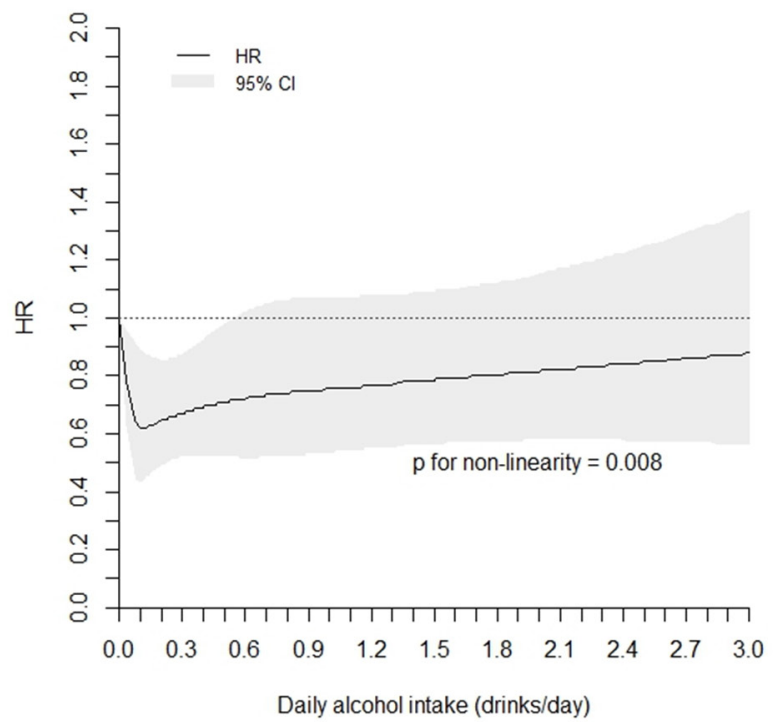

Current smokers

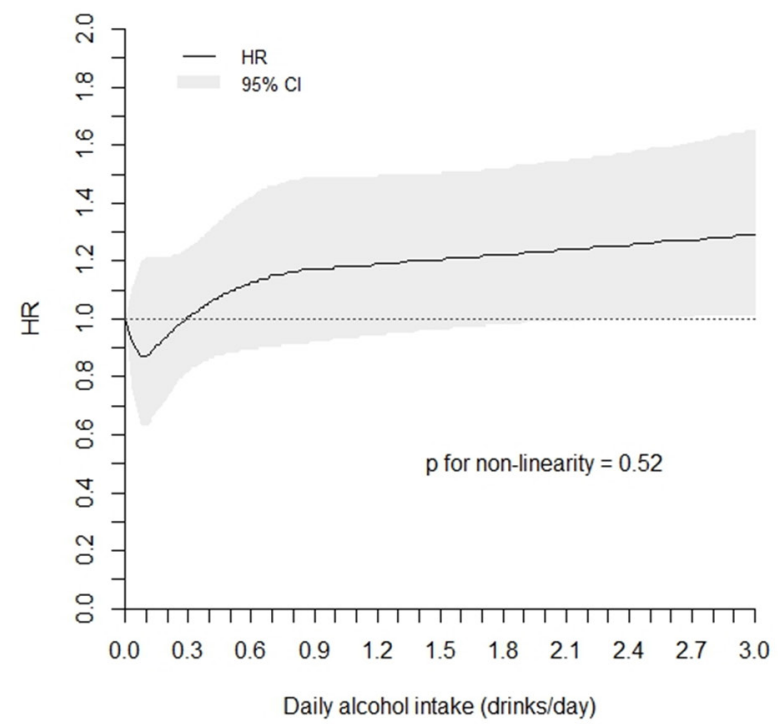

Figure 1 Association between average daily intake of alcoholic drinks and risk of active tuberculosis using restricted cubic spline regression with four knots*. *Adjusted for age at recruitment (years), year of baseline interview (1993-1995, 1996-1998), gender, dialect group (Hokkien, Cantonese), level of education (no formal education, primary school, secondary school or higher), body mass index $\left(\mathrm{kg} / \mathrm{m}^{2}\right)$, total energy intake (kcal/day), baseline history of diabetes (yes, no), tea intake (none, monthly, weekly, daily) and energy-adjusted daily intake of protein, cholesterol, marine omega-3, omega- 6 fatty acids, vitamin $A$ and vitamin C (quartiles), smoking status (never, former, current), cigarettes per day (non-current, $\leq 6,7-12,13-22,23-32$, 33-42, 43+), years of smoking (non-current smokers, $\leq 19$ years, 20-39 years, 40+ years).

Further, similar results were obtained with the exclusion of participants with less than 2 years of follow-up. We did not have information on use of immunosuppressant, but the use of systemic immunosuppressant that is sufficient to affect TB risk is unlikely to be high in a population-based cohort. The status of HIV infection among the participants is also not known, but with a very low prevalence (1278 per million population) of this condition in Singapore, ${ }^{34}$ any confounding effect from not adjusting for HIV status in this cohort is likely to have negligible impact on the observed associations. We also did not know whether the participants had close contact with patients with TB. However, alcohol intake is unlikely to be different between contacts and non-contacts of patients with TB and hence not likely to be an important confounder in our analysis. As this study was conducted in a population with low alcohol intake, the results may not be generalised to other populations with higher alcohol intake. The small number of cases in some of the subgroups in our analysis could also affect the generalisability of our results. Finally, as in any observational studies, there could still be residual confounding effect of unknown or unmeasured factors on our observed association.

The present study showed that cigarette smoking was significantly associated with approximately twofold increased risk of $\mathrm{TB}$, consistent with the results from a previous meta-analysis which included 13 studies. $^{21}$ Among current smokers, TB risk increased with smoking intensity and duration in a dose-dependent manner, further supporting a possible etiological role of smoking in the reactivation of latent TB. In this study, individuals who had quit smoking for more than 2 years before enrolment experienced similar risk as never smokers and this is consistent with findings from other studies that suggest smoking cessation to have quick improvement on airway inflammation, respiratory symptoms and bronchial hyper-responsiveness and preventing excessive decline in lung function. ${ }^{35}$ However, whether such improvements could be linked to the progression of TB disease remains to be determined.

Although heavy alcohol use or alcohol use disorder has been established to be a risk factor for active $\mathrm{TB},{ }^{6-8}$ few epidemiological studies have examined lower levels of alcohol intake in relation to TB risk. To our knowledge, the association between low levels of alcohol intake and risk of active TB has never been examined prospectively in a population-based cohort and we are the first study to report the finding that a low-dose alcohol consumption (monthly to weekly) may reduce the risk of developing active $\mathrm{TB}$, especially for never and former smokers. Low to moderate dose alcohol consumption has been reported to be beneficial to both the innate and adaptive immune system ${ }^{93} 37$ and an experimental study has shown that while a high dose of ethanol led to diminished immune responses, low dose of ethanol can improve host response to mycobacterial infection. ${ }^{15}$ Hence, the effect of alcohol intake on the immune system may strongly depend on the amount of alcohol consumed. In support of this hypothesis, the 
beneficial effects of low-dose alcohol on TB risk diminished with higher level of drinking among non-smokers in our study.

Furthermore, significant increased risk of active TB was also observed in current smokers and heavy drinkers of at least 2 drinks a day, suggesting a synergistic effect between smoking and alcohol in relation to TB disease. Oxidative metabolism of ethanol leads to the formation of acetaldehyde and/or reactive oxygen species, and oxidative stress and suppressed immunity associated with chronic alcohol consumption have been suggested as underlying mechanisms for the increased risk of developing pulmonary infection and alcoholic lung diseases. ${ }^{38}$ Acetaldehyde is also a constituent of tobacco smoke ${ }^{39}$ and the potential synergistic effect of alcohol drinking and tobacco smoking has been shown in a study in which the increase in salivary acetaldehyde concentrations after ethanol consumption was markedly higher in smokers with active smoking compared with non-smokers. ${ }^{24}$ Oxidative stress-induced lipid peroxidation in the lungs as a result of ethanol metabolism or cigarette smoke exposure can also lead to the formation of malondialdehyde. While smoking and alcohol alone can elevate both acetaldehyde and malondialdehyde levels, an experimental study conducted using mouse models has found that only the combined exposure of smoke and alcohol led to the formation of malondialdehyde-acetaldehyde adducts, ${ }^{25}$ which are very stable protein adducts capable of inducing proinflammatory response in the lungs. ${ }^{40}$ Hence, coexposure of alcohol and cigarette smoke could potentially augment lung injury via malondialdehyde-acetaldehyde adduct formation and this could explain the synergistic effect between drinking and smoking in elevating the risk of active TB in our study.

With approximately $40 \%$ of the worldwide population estimated to be current drinkers ${ }^{41}$ our findings have huge public health implications. There has been extensive literature on the benefits of moderate alcohol consumption in reducing the risk of cardiovascular diseases, ${ }^{16}$ and moderate alcohol intake (1.5-2.5 drinks/day for men and 0.5-1.5 drinks/day for women) has been increasingly promoted as part of a healthy diet to promote cardiovascular health. ${ }^{17} 18$ In our study, we have found the dose beneficial for reducing the risk of active TB to be less than one drink a day and only among non-smokers and this level of alcohol intake is lower than the level of alcohol intake recommended for cardioprotective effects. Hence the level of alcohol consumption recommended for optimum cardiovascular health may not apply to individuals with regard to their risk of developing active TB, especially current smokers. Moreover, while we report a protective association between low levels of alcohol intake in reducing the risk of active TB, further evidence is needed to validate our novel findings.

\section{CONCLUSION}

In conclusion, we observed low-dose intake of alcohol to be beneficial to never and former smokers in reducing the risk of developing active TB whereas high-dose of alcohol intake could be detrimental and act synergistically with exposure to cigarette smoke in increasing the risk of TB development. It is imperative for further studies to validate these findings before low levels of alcohol intake can be promoted as a strategy to reduce the risk of developing active TB among infected individuals.

Acknowledgements We thank Ms Siew-Hong Low of the National University of Singapore for supervising the fieldwork of the Singapore Chinese Health Study and Dr Renwei Wang for the maintenance of the cohort study database. We also thank Dr Jeffrey Cutter of the Ministry of Health and Dr Kyi-Win Khin Mar of the National Tuberculosis Notification Registry in Singapore for assistance with the identification of tuberculosis cases in the cohort.

Contributors AZS and W-PK contributed to the conception and design of the study and analysis of data. CBEC, Y-TW, J-MY and W-PK participated in the acquisition of data. AZS, CBEC, Y-TW, J-MY and W-PK were involved in the interpretation of the data and drafting of intellectual content. All authors read and approved the final manuscript and W-PK was responsible for the integrity of the work as a whole. W-PK is the guarantor.

Funding This work was supported by the United States National Cancer Institute National Institutes of Health (grant numbers UM1 CA182876 and R01 CA144034). W-PK is supported by the National Medical Research Council, Singapore (NMRC/ CSA/0055/2013). The sponsors have no role in: the study design; the collection, analysis or interpretation of data; the writing of the report or in the decision to submit the article for publication

Competing interests All authors have completed the ICMJE uniform disclosure form at and declare: support from the National Institutes of Health for the submitted work; no financial relationships with any organisations that might have an interest in the submitted work in the previous three years; no other relationships or activities that could appear to have influenced the submitted work.

Ethics approval The Institutional Review Boards of the National University of Singapore and University of Pittsburgh approved the conduct of the SCHS. The present study was approved by the Institutional Review Board of the National University of Singapore. All participants gave informed consent.

Provenance and peer review Not commissioned; internally peer reviewed.

\section{Data sharing statement № additional data available.}

Open Access This is an Open Access article distributed in accordance with the Creative Commons Attribution Non Commercial (CC BY-NC 4.0) license, which permits others to distribute, remix, adapt, build upon this work non-commercially, and license their derivative works on different terms, provided the original work is properly cited and the use is non-commercial. See: http://creativecommons.org/ licenses/by-nc/4.0/

(C) Article author(s) (or their employer(s) unless otherwise stated in the text of the article) 2017. All rights reserved. No commercial use is permitted unless otherwise expressly granted.

\section{REFERENCES}

1. World Health Organization. Global Tuberculosis Report. 2013.

2. Houben RM, Dodd PJ. The global burden of latent tuberculosis infection: a re-estimation using mathematical modelling. PLoS Med 2016;13:e1002152.

3. Lawn SD, Zumla Al. Tuberculosis. Lancet 2011;378:57-72.

4. Selwyn PA, Hartel D, Lewis VA, et al. A prospective study of the risk of tuberculosis among intravenous drug users with human immunodeficiency virus infection. N Engl J Med 1989;320:545-50.

5. Tufariello JM, Chan J, Flynn JL. Latent tuberculosis: mechanisms of host and bacillus that contribute to persistent infection. Lancet Infect Dis 2003;3:578-90.

6. Lönnroth K, Williams BG, Stadlin S, et al. Alcohol use as a risk factor for tuberculosis - a systematic review. BMC Public Health 2008;8:289.

7. Rehm J, Samokhvalov AV, Neuman MG, et al. The association between alcohol use, alcohol use disorders and tuberculosis (TB). A systematic review. BMC Public Health 2009;9:450. 
8. Imtiaz S, Shield KD, Roerecke M, et al. Alcohol consumption as a risk factor for tuberculosis: meta-analyses and burden of disease. Eur Respir J 2017:50:1700216.

9. Pasala S, Barr T, Messaoudi I. Impact of alcohol abuse on the adaptive immune system. Alcohol Res 2015;37:185-97.

10. Szabo G, Saha B. Alcohol's effect on host defense. Alcohol Res 2015;37:159-70.

11. Zhang P, Bagby GJ, Happel Kl, et al. Alcohol abuse, immunosuppression, and pulmonary infection. Curr Drug Abuse Rev 2008;1:56-67.

12. Mason CM, Dobard E, Zhang P, et al. Alcohol exacerbates murine pulmonary tuberculosis. Infect Immun 2004;72:2556-63.

13. Romeo J, Wärnberg J, Díaz LE, et al. Effects of moderate beer consumption on first-line immunity of healthy adults. J Physio Biochem 2007;63:153-9.

14. Romeo J, Wärnberg J, Nova E, et al. Changes in the immune system after moderate beer consumption. Ann Nutr Metab 2007;51:359-66.

15. Mendenhall CL, Theus SA, Roselle GA, et al. Biphasic in vivo immune function after low- versus high-dose alcohol consumption. Alcohol 1997;14:255-60.

16. Nova E, Baccan GC, Veses A, et al. Potential health benefits of moderate alcohol consumption: current perspectives in research. Proc Nutr Soc 2012;71:307-15.

17. Akbaraly TN, Ferrie JE, Berr $\mathrm{C}$, et al. Alternative healthy eating index and mortality over 18 y of follow-up: results from the Whitehall II cohort. Am J Clin Nutr 2011;94:247-53.

18. Fung $T$, McCullough ML, Newby PK, et al. Diet-quality scores and plasma concentrations of markers of inflammation and endothelial dysfunction. Am J Clin Nutr 2005;82:163-73.

19. Thomas A, Gopi PG, Santha T, et al. Predictors of relapse among pulmonary tuberculosis patients treated in a DOTS programme in South India. Int J Tuberc Lung Dis 2005;9:556-61.

20. Crampin AC, Glynn JR, Floyd S, et al. Tuberculosis and gender: exploring the patterns in a case control study in Malawi. Int $J$ Tuberc Lung Dis 2004;8:194-203.

21. Bates MN, Khalakdina A, Pai M, et al. Risk of tuberculosis from exposure to tobacco smoke: a systematic review and meta-analysis. Arch Intern Med 2007;167:335-42.

22. Koh WP, Yuan JM, Sun CL, et al. Middle-aged and older Chinese men and women in Singapore who smoke have less healthy diets and lifestyles than nonsmokers. J Nutr 2005;135:2473-7.

23. Thornton A, Lee P, Fry J. Differences between smokers, exsmokers, passive smokers and non-smokers. J Clin Epidemiol 1994:47:1143-62.

24. Salaspuro V, Salaspuro M. Synergistic effect of alcohol drinking and smoking on in vivo acetaldehyde concentration in saliva. Int $J$ Cancer 2004;111:480-3.

25. McCaskill ML, Kharbanda KK, Tuma DJ, et al. Hybrid malondialdehyde and acetaldehyde protein adducts form in the lungs of mice exposed to alcohol and cigarette smoke. Alcohol Clin Exp Res 2011;35:1106-13.
26. Ministry of Health. Enhancing public health measures against tuberculosis. Singapore, 2008.

27. Hankin JH, Stram DO, Arakawa K, et al. Singapore Chinese health study: development, validation, and calibration of the quantitative food frequency questionnaire. Nutr Cancer 2001;39:187-95.

28. Chee CB, James $L$. The Singapore tuberculosis elimination programme: the first five years. Bull World Health Organ 2003:81:217-21.

29. Gupta KB, Gupta R, Atreja A, et al. Tuberculosis and nutrition. Lung India 2009;26:9-16.

30. Patra J, Jha P, Rehm J, et al. Tobacco smoking, alcohol drinking, diabetes, low body mass index and the risk of self-reported symptoms of active tuberculosis: individual participant data (IPD) meta-analyses of 72,684 individuals in 14 high tuberculosis burden countries. PLoS One 2014:9:e96433.

31. Soh AZ, Chee CB, Wang YT, et al. Dietary cholesterol increases the risk whereas PUFAs reduce the risk of active tuberculosis in Singapore Chinese. J Nutr 2016;146:1093-100.

32. Soh AZ, Chee CBE, Wang YT, et al. Dietary intake of antioxidant vitamins and carotenoids and risk of developing active tuberculosis in a prospective population-based cohort study. Am J Epidemiol 2017; $186: 491-500$

33. Soh AZ, Pan A, Chee CBE, et al. Tea drinking and its association with active tuberculosis incidence among middle-aged and elderly adults: the Singapore Chinese health study. Nutrients 2017;9:544.

34. Ministry of Health. Communicable diseases surveillance in Singapore 2014. Ministry of Health Communicable Diseases Division, 2014:120-45.

35. Willemse BW, Postma DS, Timens W, et al. The impact of smoking cessation on respiratory symptoms, lung function, airway hyperresponsiveness and inflammation. Eur Respir $J$ 2004;23:464-76.

36. Romeo J, Wärnberg J, Nova E, et al. Moderate alcohol consumption and the immune system: a review. Br J Nutr 2007;98(Suppl 1):S111-5.

37. Barr T, Helms C, Grant K, et al. Opposing effects of alcohol on the immune system. Prog Neuropsychopharmacol Biol Psychiatry 2016:65:242-51.

38. Kaphalia L, Calhoun WJ. Alcoholic lung injury: metabolic, biochemical and immunological aspects. Toxicol Lett 2013:222:171-9.

39. Salaspuro M. Interrelationship between alcohol, smoking, acetaldehyde and cancer. Novartis Found Symp 2007;285:80-9. discussion 89-96, 198-9. [Epub ahead of print 27 jun 2017].

40. Wyatt TA, Kharbanda KK, McCaskill ML, et al. Malondialdehydeacetaldehyde-adducted protein inhalation causes lung injury. Alcohol 2012;46:51-9.

41. World Health Organization. Global status report on alcohol and health. 2014;2014:28-43. 